\title{
Comparisons among the complete genomes of four betanodavirus genotypes
}

\author{
Yasushi Okinaka*, Toshihiro Nakai \\ Graduate School of Biosphere Science, Hiroshima University, Higashi-Hiroshima 739-8528, Japan
}

\begin{abstract}
Betanodaviruses, the causative agents of viral nervous necrosis in marine fish, have bipartite positive-sense RNA genomes and have been classified (based on analysis of RNA2 sequences) into 4 genotypes: tiger puffer nervous necrosis virus (TPNNV), barfin flounder nervous necrosis virus (BFNNV), striped jack nervous necrosis virus (SJNNV), and redspotted grouper nervous necrosis virus (RGNNV). Full-length genomes of TPNNV and BFNNV were sequenced for the first time in this study. Their sequence data and those of SJNNV and RGNNV retrieved from GenBank were compared in order to investigate the relationships among the 4 genotypes. Between TPNNV and BFNNV, sequence identities were relatively high in RNA1 and encoded Protein A, but were not significantly high in RNA2 or the coat protein (CP). Similarly, between BFNNV and RGNNV, the amino acid sequences of CP were highly similar, but identities of RNA1, RNA2, and Protein A sequences were not especially high. Furthermore, multiple alignment data of the 4 genotypes of RNA2 sequences revealed that the TPNNV and SJNNV sequences have the same sizes of gaps and extra sequences at the same positions. Collectively, these apparent contradictions in sequence identity suggest that betanodavirus genomes have been constructed via complex evolutionary processes.
\end{abstract}

KEY WORDS: Betanodavirus · Complete genomic sequence $\cdot$ RNA1 RNA2 - Tiger puffer nervous necrosis virus $\cdot$ Barfin flounder nervous necrosis virus

\section{INTRODUCTION}

Betanodaviruses are the causative agents of a highly destructive disease of hatchery-reared larvae and juveniles of a wide variety of marine fish. In some species, adult and mature fish have also been reported to suffer from the disease. The disease, designated viral nervous necrosis (VNN) when it was first described in 1990 (Yoshikoshi \& Inoue 1990), is also known as viral encephalopathy and retinopathy (Office International des Epizooties 2006). VNN has spread to more than 30 marine fish species from 14 families in the IndoPacific and Mediterranean regions, Scandinavia, and North America. The virus localizes in the brain, spinal cord, and retina of the affected fish. Affected fish exhibit erratic swimming patterns and a range of neurological abnormalities, including vacuolization and cellular necrosis in the central nervous system and retina (Munday et al. 2002).

Betanodaviruses, members of the family Nodaviridae, are nonenveloped, spherical viruses with a bipar- tite positive-sense RNA genome consisting of RNA1 (3.1 kb) and RNA2 (1.4 kb), which encode an RNAdependent RNA polymerase (Protein A) and the coat protein (CP), respectively (Schneemann et al. 2005). Recently, we characterized a subgenomic RNA3 (0.4 kb), which encodes Protein B2, a suppressor for post-transcriptional gene silencing (Iwamoto et al. 2005). These characteristics are similar to those of the insect alphanodaviruses (Ball \& Johnson 1998, Schneemann et al. 2005) belonging to the other genus of the family Nodaviridae. Based on similarities in the partial RNA2 sequences (so-called T4 region sequences), betanodaviruses have been classified into 4 genotypes, designated striped jack nervous necrosis virus (SJNNV), barfin flounder nervous necrosis virus (BFNNV), tiger puffer nervous necrosis virus (TPNNV), and redspotted grouper nervous necrosis virus (RGNNV) (Nishizawa et al. 1997). Recently, a betanodavirus isolate from turbot Scophthalmus maximus (TNV) was suggested to belong to a fifth genotype (Johansen et al. 2004). In addition, most of the 21 
betanodavirus isolates from France, Spain, Tunisia, and Tahiti were classified as RGNNV, and were further divided into 2 distinct subtypes (Thiéry et al. 2004). Phylogenetic analysis of betanodaviruses has been commonly performed using the T4 sequences. However, in some studies, the T2 region (Nishizawa et al. 1994), which covers a larger RNA2 sequence than T4, is also utilized for phylogenetic analysis (e.g. Grotmol et al. 2000, Chi et al. 2003, Johansen et al. 2004). Clinical disease caused by SJNNV and TPNNV has only been reported in striped jack Pseudocaranx dentex and tiger puffer Takifugu rubripes, respectively. However, recently, SJNNV was found in European sea bass Dicentrarchus labrax, sea bream Sparus aurata and Senegalese sole Solea senegalensis farmed in the Iberian Peninsula - though the samples used were from subclinically infected fish (Thiéry et al. 2004, Cutrín et al. 2007). BFNNV has been isolated from some coldwater species, such as barfin flounder Verasper moseri, Pacific cod Gadus macrocephalus, and Atlantic halibut Hippoglossus hippoglossus. RGNNV has a broad host range and causes disease among a variety of warm-water fish species, particularly groupers and sea bass (Munday et al. 2002).

As mentioned earlier, betanodavirus isolates have been characterized based on similarities in the partial RNA2 sequences. Thus, not much is known about the RNA1 sequences and the remaining RNA2 region. So far, complete nucleotide sequences of both genomic segments have been determined for 1 SJNNV isolate (Iwamoto et al. 2001) and 4 RGNNV isolates (Tan et al. 2001, Iwamoto et al. 2004, M. W. Lu, T. C. Guo \& J. Wu unpubl. data, X. Chen, J. He \& J. Huang unpubl. data, X. Z. Chen, Y. Q. Gong \& Y. Q. Su unpubl. data) isolates. For BFNNV, only the RNA1 segment has been sequenced completely (Sommerset \& Nerland 2004). Neither of the genomic segments has been fully sequenced for TPNNV. Determining the entire genomic sequences of all 4 genotypes of betanodaviruses should give better insights into the relationships among them. In this study, we determined whole genomic sequences of TPNNV and BFNNV isolates for the first time to compare the data with other full-length betanodavirus sequences.

\section{MATERIALS AND METHODS}

Preparation of viruses. TPKag93 (TPNNV genotype) and JFIwa98 (BFNNV genotype) isolates (Iwamoto et al. 1999) were inoculated separately into E-11 cells (Iwamoto et al. 2000) and the cells were cultured at $20^{\circ} \mathrm{C}$ for $5 \mathrm{~d}$ in Leibovitz's L-15 medium (Invitrogen) supplemented with $5 \%$ fetal bovine serum. Progeny viruses were collected from the culture supernatants and concentrated as described in Furusawa et al. (2006).

Determination of TPKag93 and JFIwa98 genomic RNA sequences. TPKag93 and JFIwa98 genomic RNAs were extracted separately from the purified virions by using ISOGEN-LS (Nippon Gene) according to the manufacturer's instructions. To obtain initial viral cDNA clones, double-stranded cDNA was synthesized from the extracted RNA of each virus using the SuperScript Choice System for cDNA Synthesis kit (Invitrogen) and random hexamer oligonucleotide primers according to the supplier's instructions. cDNA thus obtained was cloned into pBluescript SK (-) (Stratagene) and large cDNA clones for RNA1 and RNA2 were selected by PCR using M4 and RV primers (Table 1) that amplify a cloned DNA fragment in this vector. Viral cDNA sequences were determined to confirm the identities of 2 independent clones for both RNA1 and RNA2. Since obtained cDNA clones seemed to lack $5^{\prime}$ and $3^{\prime}$ end sequences, terminal sequences were determined by the rapid amplification of cDNA ends (RACE) method as described in Iwamoto et al. (2001). Briefly, for 5' RACE, the extracted viral RNA was reverse-transcribed using TP1-R1 or TP2-R1 for TPKag93 and BF1-R1 or BF2-R1 for JFIwa98 (Table 1). The first-strand cDNAs were polyadenylated with poly(A) polymerase and the second-strand cDNAs were synthesized using the ANCH primer (Table 1). The double-stranded cDNAs were amplified using the reverse primers TP1-R2 or TP2-R2 for TPKag93 and BF1-R2 or BF2-R2 for JFIwa98, along with AUAP as the forward primer (Table 1). For 3' RACE, the viral RNA was polyadenylated as described above and then reverse-transcribed using the ANCH primer (Table 1). The first-strand cDNAs were amplified with the forward primers TP1-F1 or TP2-F1 for TPKag93 and BF1F1 or BF2-F1 for JFIwa98, together with AUAP as the reverse primer (Table 1). The amplified products were purified using QIAquick PCR Purification Kit (QIAGEN) and further amplified by nested PCR using the forward primers TP1-F2 or TP2-F2 for TPKag93 and BF1-F2 or BF2-F2 for JFIwa98, along with AUAP (Table 1). The PCR products thus obtained in 3' RACE and $5^{\prime}$ RACE were used to determine the terminal sequences.

Comparative analyses of viral sequences. All the full-length sequences of betanodavirus RNA1 and RNA2 so far deposited in GenBank were retrieved. Their sequence IDs and virus genotypes are listed in Table 2. Viral RNA sequences and deduced amino acid sequences were assembled and analyzed using the ClustalW program (www.ebi.ac.uk/Tools/clustalw/). Alphanodavirus sequences (Table 2) were also retrieved from GenBank to compare their sizes with those of the betanodaviruses. 
Table 1. Oligonucleotide primers used in this study

\begin{tabular}{|lll|}
\hline Name & \multicolumn{1}{c|}{ Sequence $\left(5^{\prime}-3^{\prime}\right)$} & \\
\hline ANCH & GGCCACGCGTCGACTAGTACTTTTTTTTTTTTTTTTTT & 5' RACE and 3' RACE \\
AUAP & GGCCACGCGTCGACTAGTAC & 5' RACE and 3' RACE \\
TP1-F1 & CAAACAGACAAGGATGGAAC & 3' RACE for TPKag93 RNA1 \\
TP1-F2 & CTCTTCCAGGCGTTGATGGA & 3' RACE for TPKag93 RNA1 \\
TP2-F1 & CTAGTGCGTATCGTTGATGAC & 3' RACE for TPKag93 RNA2 \\
TP2-F2 & GACATTGAAGCTATCGCTAAC & 3' RACE for TPKag93 RNA2 \\
TP1-R1 & ATGGCTCGTAGCCACTTTC & 5' RACE for TPKag93 RNA1 \\
TP1-R2 & AACGCTCAGCATGGTTCAG & 5' RACE for TPKag93 RNA1 \\
TP2-R1 & TGGATCAGGCAGGAAGC & 5' RACE for TPKag93 RNA2 \\
TP2-R2 & CACATTGGCTGAATGTCGAACTC & 5' RACE for TPKag93 RNA2 \\
BF1-F1 & CAAACTGACAAGGATGGAACA & 3' RACE for JFIwa98 RNA1 \\
BF1-F2 & CTCTTCCAGGTGTTGATGGA & 3' RACE for JFIwa98 RNA1 \\
BF2-F1 & CTGGTGCGTATCGTTGATGA & 3' RACE for JFIwa98 RNA2 \\
BF2-F2 & GACATTGAAGCTATCGCTAAC & 3' RACE for JFIwa98 RNA2 \\
BF1-R1 & GAGGCGATGGAGAAATGATGT & 5' RACE for JFIwa98 RNA1 \\
BF1-R2 & AACGCTCAACATGGTTTCAG & 5' RACE for JFIwa98 RNA1 \\
BF2-R1 & TGGATCAGGCAGGAAGC & 5' RACE for JFIwa98 RNA2 \\
BF2-R2 & CTCAACAGCGTATCGCTGGAAG & 5' RACE for JFIwa98 RNA2 \\
M4 & GTTTTCCCAGTCACGAC & Amplification of a clone in pBluescript SK (-) \\
RV & CAGGAAACAGCTATGAC & Amplification of a clone in pBluescript SK (-) \\
\hline
\end{tabular}

Table 2. IDs of betanodavirus RNA sequences used in this study. Virus names are abbreviated in this study for convenience of explanation: WSNNV: white star snapper nervous necrosis virus; ECNNV: Epinephelus coioides nervous necrosis virus; DGNNV: dragon grouper nervous necrosis virus. -: no sequence available; ND: not designated. RNA1, RNA2: GenBank accession numbers of RNA1 and RNA2 sequences, respectively

\begin{tabular}{|lcccl}
\hline Isolate & Genotype & \multirow{2}{*}{ RNA1 } & \multirow{2}{*}{ RNA2 } & \multicolumn{1}{c|}{ Reference } \\
\hline \multicolumn{2}{l}{ Betanodavirus } & & & \\
TPKag93 & TPNNV & EU236148 & EU236149 & This study \\
JFIwa98 & BFNNV & EU236146 & EU236147 & This study \\
AHNV & BFNNV & AJ401165 & - & Sommerset \& Nerland (2004) \\
SJNag93 & SJNNV & AB056571 & AB056572 & Iwamoto et al. (2001) \\
SGWak97 & RGNNV & AY324869 & AY324870 & Iwamoto et al. (2004) \\
GGNNV & RGNNV & AF319555 & AF318942 & Tan et al. (2001) \\
WSNNV & RGNNV & - & AY835642 & M. W. Lu et al. (unpubl. data) \\
ECNNV & RGNNV & AY369136 & AF534998 & X. Chen et al. (unpubl. data) \\
DGNNV & RGNNV & AY721616 & AY721615 & X. Z. Chen et al. (unpubl. data) \\
TNV & ND & - & AJ608266 & Johansen et al. (2004) \\
Alphanodavirus & & & \\
FHV & ND & X77156 & X15959 & Dasgupta \& Sgro (1989) \\
BBV & ND & X02396 & X00956 & Dasmahapatra et al. (1985) \\
BoV & ND & AF329080 & - & Johnson et al. (2001) \\
PaV & ND & AF171942 & AF171943 & Johnson et al. (2000) \\
NoV & ND & AF174533 & AF174534 & Johnson et al. (2003) \\
\hline
\end{tabular}

ments so far deposited in GenBank were retrieved and are summarised in Table 3. Determination of TPKag93 and JFIwa98 sequences allowed us to compare the genomes of 4 betanodavirus genotypes. Among the betanodaviruses, RNA1s varied in length from $3100 \mathrm{nt}$ for Atlantic halibut nodavirus (AHNV) to 3112 nt for TPKag93 and encoded Protein A contained 981 to 983 amino acids (Table 3). Similarly, RNA2s varied from $1417 \mathrm{nt}$ for TNV to $1434 \mathrm{nt}$ for SGWak97 and encoded CPs contained 338 to 340 amino acids. Betanodavirus RNA3s ranged in size from $371 \mathrm{nt}$ for AHNV to 378 nt for SJNag93 but encoded Protein B2s contained identical numbers of amino acids (75). Complete nucleotide sequences of RNA3 have been determined experimentally only for SJNag93 (Iwamoto et al. 2005) and AHNV (Sommerset \& Nerland 2004). However, RNA3 sequences of the other 6 betano-

\section{RESULTS AND DISCUSSION}

\section{Size differences of RNA segments and encoded proteins among the Nodaviridae}

Complete nucleotide sequences of TPKag93 and JFIwa98 genomes (Table 2) were determined for the first time. The lengths of their RNA segments and encoded proteins are listed in Table 3. All the full-length sequences of betanodavirus and alphanodavirus RNA seg- daviruses were predicted easily because their RNA1 portions, which included putative $5^{\prime}$ termini of RNA3 (5'UAGUCA---), were conserved and identical to the known authentic sequences of SJNag93 and AHNV (5'-UCCAAGCCGGUCCUAGUCAA-3'). Additionally, the 3 ' termini of RNA1 and RNA3 are identical for SJNag93 and AHNV. A possible initiation site for Protein B1 was not observed in any of the $5^{\prime}$-proximal regions of the betanodavirus RNA3s (data not shown). Compared to a series of differences among the betanodavirus iso- 
Table 3. Lengths of nodavirus RNA segments and encoded proteins. See Table 2 legend for virus names

\begin{tabular}{|c|c|c|c|c|c|c|}
\hline \multirow{2}{*}{ Isolate } & \multicolumn{3}{|c|}{ - Nucleotide number - } & \multicolumn{3}{|c|}{ — Amino acid number } \\
\hline & RNA1 & RNA2 & RNA3 & Protein A & $\mathrm{CP}$ & Protein B2 \\
\hline \multicolumn{7}{|c|}{ Betanodavirus } \\
\hline TPKag93 & 3112 & 1422 & $375^{\mathrm{a}}$ & 982 & $340^{\mathrm{b}}$ & 75 \\
\hline JFIwa98 & 3101 & 1433 & $372^{\mathrm{a}}$ & 981 & $338^{\mathrm{b}}$ & 75 \\
\hline AHNV & 3100 & - & 371 & 981 & - & 75 \\
\hline SJNag93 & 3107 & 1421 & 378 & $983^{\mathrm{b}}$ & $340^{\mathrm{b}}$ & $75^{\mathrm{b}}$ \\
\hline SGWak97 & 3105 & 1434 & $376^{\mathrm{a}}$ & 982 & $338^{\mathrm{b}}$ & 75 \\
\hline GGNNV & 3103 & 1433 & $374^{\mathrm{a}}$ & 982 & $338^{\mathrm{b}}$ & 75 \\
\hline WSNNV & - & 1433 & - & - & 338 & - \\
\hline ECNNV & 3103 & 1433 & $374^{\mathrm{a}}$ & 982 & 338 & 75 \\
\hline DGNNV & 3103 & 1433 & $374^{\mathrm{a}}$ & 982 & 338 & 75 \\
\hline TNV & - & 1417 & - & - & 340 & - \\
\hline \multicolumn{7}{|c|}{ Alphanodavirus } \\
\hline FHV & 3107 & 1400 & 387 & 998 & 407 & $106^{\mathrm{b}}$ \\
\hline BBV & 3106 & 1399 & 387 & 998 & 407 & $106^{\mathrm{b}}$ \\
\hline $\mathrm{BoV}$ & 3096 & - & 388 & 998 & - & $106^{\mathrm{b}}$ \\
\hline $\mathrm{PaV}$ & 3011 & 1311 & 414 & 973 & 401 & $90^{\mathrm{b}}$ \\
\hline NoV & 3204 & 1336 & 473 & 1042 & 399 & 137 \\
\hline \multicolumn{7}{|c|}{$\begin{array}{l}\text { aNucleotide numbers were predicted based on known RNA3 sequences } \\
\text { (Iwamoto et al. 2004, Sommerset \& Nerland 2004) } \\
\text { bProtein synthesis has been confirmed }\end{array}$} \\
\hline
\end{tabular}

lates, the sizes of RNAs and encoded proteins in alphanodaviruses were more varied (Table 3), indicating that betanodaviruses are phylogenetically closer to each other than alphanodaviruses. This observation is supported by the fact that the Protein A and CP sequences of Flock House virus (FHV), black beetle virus (BBV), Boolarra virus (BoV), Pariacoto virus $(\mathrm{PaV})$, and Nodamura virus (NoV) shared 26 to $99 \%$ and 39 to $87 \%$ identities to each other, respectively (Johnson et al. 2000, 2001). These identities are more varied than those of the betanodaviruses obtained in this study (Fig. 1). Moreover, some alphanodaviruses translate minor proteins from RNA1 (FHV and BBV, Harper 1994) and RNA2 (PaV, Johnson \& Ball 2003), but no minor protein has yet been detected in betanodaviruses. Finally, NoV is the only nodavirus that has been shown to infect warmblooded animals (suckling mice, Ball \& Johnson 1998). These divergences in alphanodaviruses suggest that genetic variations of betanodaviruses will increase in the future. Alternatively, alphanodaviruses and betanodaviruses could be genetically distant though they both belong to the same family.

\section{Homology in RNA1 and Protein A among betanodaviruses}

Homology of RNA1 and Protein A sequences among the 4 genotypes of betanodaviruses were evaluated by pairwise comparisons (Fig. 1). Among the 8 betanodavirus isolates used in this study, the Protein A sequences were significantly similar to each other
(87 to $99 \%$ identities). The C-termini of the Protein A sequences were arginine-rich and were relatively less identical to each other (data not shown). Likewise, the RNA1 sequences had 82 to $98 \%$ shared RNA1 sequence identities with each other. The levels of shared RNA1 sequence identities were especially high between TPKag93 and the 2 BFNNV isolates (JFIwa98 and AHNV) (91\%), though TPKag93 and the latter 2 viruses belonged to different genotypes. This may be attributed to the limited range of temperatures (15 to $20^{\circ} \mathrm{C}$ ) under which TPKag93 and JFIwa98 can multiply (Iwamoto et al. 2000), compared to the wider range of temperatures under which RGNNV isolates can multiply ( 15 to $35^{\circ} \mathrm{C}$ ) (Hata et al. 2007, authors' unpubl. data). We found Protein A to be the predominant control of betanodavirus temperature sensitivity (authors' unpubl. data). The 5' untranslated regions (UTRs) of RNA1s in the 8 betanodaviruses were highly homologous to each other, but the 5' UTR of SJNag93 had relatively low levels of shared identities with those of the RGNNV isolates, SGWak97 $(83 \%)$, greasy grouper nervous necrosis virus (GGNNV, 80\%), Epinephelus coioides nervous necrosis virus (ECNNV, 82\%), and dragon grouper nervous necrosis virus (DGNNV, 82\%). The multiple alignment of the eight $5^{\prime}$ UTRs of RNA1s revealed that TPKag93 had the longest sequence (86 nt) among the viruses and gave large gaps of 7 to 8 consecutive nucleotides in the other isolates (Fig. 2A). However, the RNA1 5' UTR sequences were highly similar between TPKag93 and the 2 BFNNV isolates (JFIwa98 and AHNV) (Fig. 1). Likewise, the RNA1 3' UTRs were highly homologous between TPKag93 and the 2 BFNNV isolates (93 to 94\%) (Fig. 1). In contrast, the RNA1 3' UTRs of JFIwa98 and AHNV had less than $78 \%$ shared identities with those of the RGNNV isolates (SGWak97, GGNNV, ECNNV, and DGNNV). These low levels of RNA1 3' UTR identities shared between the BFNNV and RGNNV isolates were contradictory to their moderate levels of shared RNA1 5' UTR and Protein A identity, and to their high levels of shared RNA2 and CP identities, which is mentioned in the next section. This series of apparent contradictions should give an insight into recombination and reassortment events in betanodaviruses. Curious observations were that the 3' ends of RNA1s in AHNV, GGNNV, ECNNV, and DGNNV were $1 \mathrm{nt}$ shorter than those in the other viruses (Fig. 2B). 
RNA1

\begin{tabular}{|c|c|c|c|c|c|c|c|c|c|c|}
\hline & | TPKag93 & JFlwa98 & AHNV & | SJNag93 & | SGWak97 & GGNNV & ECNNV & DGNNV & WSNNV & TNV \\
\hline TPKag93 & 工 & 91 & 91 & 82 & 82 & 82 & 82 & 82 & - & - \\
\hline $\begin{array}{l}\text { JFIwa98 } \\
\end{array}$ & 95 & & 98 & 83 & 83 & 83 & 82 & 83 & - & - \\
\hline AHNV & 95 & 99 & & 83 & 83 & 83 & 82 & 83 & - & - \\
\hline SJNag93 & 87 & 87 & 88 & & 82 & 82 & 82 & 82 & - & - \\
\hline SGWak97 & 88 & 88 & 88 & 87 & & 97 & 96 & 98 & - & - \\
\hline GGNNV & 88 & 88 & 88 & 87 & 98 & & 96 & 97 & - & - \\
\hline ECNNV & 88 & 88 & 88 & 87 & 98 & 98 & & 96 & - & - \\
\hline DGNNV & 89 & 88 & 89 & 88 & 99 & 99 & 98 & & - & - \\
\hline WSNN & - & - & - & - & - & - & - & - & & - \\
\hline TNV & - & - & - & - & - & - & - & - & - & \\
\hline
\end{tabular}

RNA1 5' UTR

\begin{tabular}{|c|c|c|c|c|c|c|c|c|c|c|}
\hline & | TPKag93 & JFIwa98 & AHNV & |SJNag93 & SGWak97 & GGNNV & ECNNV & DGNNV & WSNNV & TNV \\
\hline TPKag93 & ( & 93 & 93 & 87 & 88 & 85 & 85 & 87 & - & - \\
\hline JFIwa98 & 94 & & 100 & 87 & 88 & 85 & 87 & 87 & - & - \\
\hline AHNV & 93 & 98 & & 87 & 88 & 85 & 87 & 87 & - & - \\
\hline SJNag93 & 90 & 85 & 86 & & 83 & 80 & 82 & 82 & - & - \\
\hline$\overline{\text { SGWak97 }}$ & 80 & 76 & 77 & 85 & & 97 & 97 & 98 & - & - \\
\hline GGNNV & 80 & 75 & 76 & 81 & 97 & & 97 & 98 & - & - \\
\hline ECNNV & 81 & 76 & 77 & 82 & 98 & 98 & & 98 & - & - \\
\hline DGNNV & 82 & 77 & 78 & 84 & 100 & 97 & 98 & & - & - \\
\hline WSNN & - & - & - & - & - & - & - & - & & - \\
\hline TNV & - & - & - & - & - & - & - & - & - & \\
\hline
\end{tabular}

RNA2

\begin{tabular}{|c|c|c|c|c|c|c|c|c|c|c|}
\hline & | TPKag93 | & JFIwa98 & AHNV & | SJNag93 & SGWak97 & GGNNV & ECNNV & DGNNV & WSNNV & TNV \\
\hline TPKag93 & & 81 & - & 82 & 81 & 80 & 81 & 81 & 81 & 77 \\
\hline JFlwa98 & 81 & & - & 81 & 82 & 82 & 82 & 82 & 82 & 78 \\
\hline ANHV & - & - & & - & - & - & - & - & - & - \\
\hline SJNag93 & 80 & 81 & - & & 80 & 79 & 80 & 80 & 79 & 76 \\
\hline SGWak97 & 81 & 86 & - & 81 & & 99 & 99 & 98 & 98 & 78 \\
\hline GGNNV & 81 & 86 & - & 81 & 98 & & 98 & 98 & 98 & 78 \\
\hline ECNNV & 81 & 86 & - & 81 & 99 & 99 & & 98 & 99 & 78 \\
\hline DGNNV & 81 & 86 & - & 81 & 99 & 99 & 100 & & 99 & 78 \\
\hline WSNNV & 81 & 86 & - & 81 & 99 & 98 & 99 & 98 & & 78 \\
\hline TNV & 78 & 80 & - & 77 & 78 & 78 & 78 & 78 & 78 & \\
\hline
\end{tabular}

RNA2 5' UTR

\begin{tabular}{|c|c|c|c|c|c|c|c|c|c|c|}
\hline & |TPKag93 & | JFlwa98 & AHNV & SJNag93 & SGWak97 & GGNNV & ECNNV & DGNNV & WSNNV & TNV \\
\hline TPKag93 & & 61 & - & 70 & 61 & 61 & 61 & 61 & 61 & 85 \\
\hline JFlwa98 & 87 & & - & 69 & 88 & 88 & 88 & 88 & 88 & 80 \\
\hline ANHV & - & - & & - & - & - & - & - & - & - \\
\hline SJNag93 & 86 & 88 & - & & 73 & 73 & 73 & 73 & 73 & 55 \\
\hline SGWak97 & 84 & 82 & - & 83 & 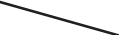 & 100 & 100 & 100 & 100 & 88 \\
\hline GGNNV & 84 & 82 & - & 83 & 99 & & 100 & 100 & 100 & 88 \\
\hline ECNNV & 84 & 81 & - & 83 & 99 & 99 & & 100 & 99 & 88 \\
\hline DGNNV & 85 & 82 & - & 84 & 98 & 98 & 98 & & 99 & 88 \\
\hline WSNNV & 84 & 82 & - & 83 & 99 & 99 & 100 & 100 & & 88 \\
\hline TNV & 81 & 85 & - & 84 & 81 & 80 & 81 & 81 & 80 & \\
\hline
\end{tabular}

\begin{tabular}{|c|c|c|c|c|c|c|c|c|c|c|}
\hline & & & & & RNA3 & & & & & \\
\hline & |TPKag93 & JFlwa98 & AHNV & SJNag93 & | SGWak97 & GGNNV & ECNNV & DGNNV & WSNNV & TNV \\
\hline TPKag93 & - & 91 & 91 & 82 & 82 & 83 & 82 & 83 & $\begin{array}{l}- \\
-\end{array}$ & - \\
\hline JFIwa98 & 90 & & 99 & 85 & 82 & 82 & 82 & 83 & - & - \\
\hline AHNV & 90 & 100 & & 85 & 83 & 83 & 83 & 83 & - & - \\
\hline $\begin{array}{l}\text { SJNag93 } \\
\end{array}$ & 77 & 78 & 78 & & 81 & 82 & 82 & 82 & - & - \\
\hline SGWak97 & 78 & 82 & 82 & 73 & 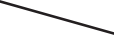 & 97 & 95 & 98 & - & - \\
\hline GGNNV & 81 & 85 & 85 & 74 & 97 & & 97 & 98 & - & - \\
\hline ECNNV & 81 & 84 & 84 & 76 & 94 & 97 & & 96 & - & - \\
\hline DGNNV & 81 & 85 & 85 & 74 & 97 & 100 & 97 & & - & - \\
\hline WSNN & - & - & - & - & - & - & - & - & & - \\
\hline TNV & - & - & - & - & - & - & - & - & - & \\
\hline
\end{tabular}

Fig. 1. Comparisons of the RNA (UTR: untranslated region) and encoded protein (CP: coat protein) sequences among the 9 betanodavirus isolates. Nucleotide and amino acid sequences were compared using the ClustalW program. Sequence identities of $\leq 80 \%, 81-85 \%, 86-90 \%$, and $91-100 \%$ are indicated in black, blue, green, and red, respectively. '-' indicates no sequence was available. See Table 2 legend for virus names 


\section{A}

TPKag93 UAACAUUGUCU-CUUGCUCUGUUGAAUAACUGAAUAAUCACUUACGCAAGGUUACCGUUUUAGCAUACACAACGAUAAGUGUACGCOAUG JFIwa98 UAACAUUAUCU-CUUGCUCUGUUGAG-------AUA-UCACUUACGCAAGGUUACCGUUUAAGCAUACACAACGACAAGUGUAAGCOAUG AHNV UAACAUUAUCU-CUUGCUCUGUUGAG-------AUA-UCACUUACGCAAGGUUACCGUUUAAGCAUACACAACGACAAGUGUAAGCCAUG SJNag 93 UAACAUCAGCU-CUUGCUCUGUUGUUU-------UGAACACUUACGCAAGGUUACCGUUU-AGCAUACAAUACGACAAUUGUAAGCCAUG SGWak97 UAACAUCACCUUCUUGCUCUGUUGAG--------UAAUCACUUACGCAAGGUUACCGUUU-AGCUUAGACAACGACAAGUCUACGCOAUG GGNNV UAACAUCACCUUCUUGCUCUGUUGAG--------UAAUCACUUACGCAAGGUUACCGUAC-AGCUUAGACAACGACAAGUCUACGCOAUG ECNNV UAACAUCACCUUCUUGCUCUGUUGAG--------UCAUCACUUACGCAAGGUUACCGUUC-AGCUUAGACAACGACAAGUCUACGCOAUG DGNNV UAACAUCACCUUCUUGCUCUGUUGAG--------UAAUCACUUACGCAAGGUUACCGUUC-AGCUUAGACAACGACAAGUCUACGCCAUG

\section{B} $* * * * * * * * * * * * * * * * * * \quad * \quad * * * * * * * * * * * * * * * * * * * \quad * * * * * * * * * * * * * * * * * *$

TPKag 93 UAGGC-AGUCACCUGCCCGCUCCCACCCCCCCCGGACCUACGGGUCCCCUAGUCAGCUUUAUGCUGUCAUACGCUUCGGCG JF Iwa98 UAGGC-AGUCACCUGCCCGCUCCCACCCCCCCCGGGCCUACGGGCCCCCUAGUCAGCUUUACGCUGUCAUACGCUUAGGCG AHNV UAGGC-AGUCACCUGCCCGCUCCCACCCCCCCCGGGCCUAUGGGCCCCCUAGUCAGCUUUACGCUGUCAUACGCUUAGGCSJNag 93 UAGGCGAGUCACCUGCCCGCUCCUACCCCCCCCGGACC-AUUGGUCCCCUAGUCAGCUUUAUGCUGUCCUACGCUUCGGCG SGWak97 UAGACAGGUCACCUGCCUGCUCCCACCCCCCUGGACCGUUUUGGUCCCUUAAUCAGCUUUAUGCUGUCCUACGCUUCGGCG GGNNV UAGACAGGUCACUUGCCUGCUCCCACCCCU-UGGACCGUUUUGGUCCCUUAAUCAGCUUUAUGCUGUCCUACGCUUCGGCECNNV UAGACAGGUCACUUGCCUGCUCCCACCCCC-UGGACCGUUUUGGUCCCUUAAUCAGCUUUAUGCUGUCCUACGCUUCGGCDGNNV UAGACAGGUCACCUGCCUGCUCCCACCCCC-UGGACCGUUUUGGUCCCUUAAUCAGCUUUAUGCUGUCCUACGCUUCGGC-

\section{C} TPKag93 UAAUCCUUCAUUGCUUUGCUU-AUCAAAAUG JFIwa98 UAAUCCAACACUGCUUUGCCA--UCACAAUG SJNag93 UAAUCUAACACCGCUUUGCAAG-UCAAAAUG SGWak97 UAAUCCAUCACCGCUUUGCAA--UCACAAUG GGNNV UAAUCCAUCACCGCUUUGCAA--UCACAAUG ECNNV UAAUCCAUCACCGCUUUGCAA--UCACAAUG DGNNV UAAUCCAUCACCGCUUUGCAA--UCACAAUG WSNNV UAAUCCAUCACCGCUUUGCAA--UCACAAUG TNV UAAUCCUUCGCCGCUUUGCUUUAUCACAAUG

(27) (26) (27) (26) (26) (26) (26) (26) (28)

\section{D}

TPKag 93 UAAGCGGGUCGUCCGGUUCCCUAGUGCGUAUCGUUGAUGACCAAUUUGACAAACUGAUUAAUGCACUAAGCACAAU--------------JF Iwa98 UAACCGGGUCAUCCGGUUCCCUGGUGCGUAUCGUUGAUGACCAAUUUGACAAAUUGAUUCAAGCACUAAGCUCCAUUAAUGCCGAAAUUG SJNag 93 UAACCGGGUCAUCCGGAUCCCUAGUGCGUAUCGUGGAUGACCAAUUCGAGAAAUUGAUUACGGCACUAACCACUAU--------------SGWak97 UAA CCGGGUCAUCCGGUUCCCUAGUGCGUAUCGUUGAUGACCAAUUUGAACAAUUGAUUAAAGCACUAACAAAUAUAAAUAAAGAAAUAC GGNNV UAACCGGGUCAUCCGGUUCCCUAGUGCGUAUCGUUGAUGACCAAUUUGAACAAUUGAUUAAAGCACUAACAAACAUAAAUAAAGAAAUAC ECNNV UAACCGGGUCAUCCGGUUCCCUAGUGCGUAUCGUUGAUGACCAAUUUGAACAAUUGAUUAAAGCACUAACAAAUAUAAAUAAAGAAAUAC DGNNV UAACCGGGUCAUCCGGUUCCCUAGUGCGUAUCGUUGAUGACCAAUUUGAACAAUUGAUUAAAGCACUAACAAAUAUAAAUAAAGAAAUAC WSNNV UAACCGGGUCAUCCGGUUCCCUAGUGCGUAUCGUUGAUGACCAAUUUGAACAAUUGAUUAAAGCACUAACAAAUAUAAAUAAAGAAAUAC TNV UAACCGGGUCAUCCGGUUCCCUGGUGCGUAUCGUUGAUGACCAAUUUAAUAAAUUAAUUGAAGCACUAACCACGAU--------------$* * * * * * * * * * * * * * * * * * * * * * * * * * * * * * * * * * * * * * * * * * \quad * * * * * * * \quad * *$

TPKag93 ------UAGAACAGAAAUUGACAAGAAUAAGAGCGACAUUGAAGCUAUCGCUAACAAAUUAAACGACAAAGCACCCAAGGAGGGUACGA JFIwa98 CUACAAACAAAACAGAAAUUGACAGAAACAAAAGCGACAUUGAAGCUAUCGCUAACAAAUUAGACAACAAAGCACCCAAGGAGGGUACAA SJNag 93 ------CAAAAUUGAAAUUGACAACAACAAGAGCGAAAUUGAAGCUAUCGCUAACAAAUUAAACGACAAAGCACCCAAGGAGGGCUCGA SGWak97 AAACAAACAAAACUGAAAUUGGAAAGAAUAGAAGCGAAAUUGAAUCACUCGCUAGCAAAUUAAACGACAAAGCACCCAAGGAGGGUGCGA GGNNV AAACAAACAAAACUGAAAUUGGAAAGAAUAGAAGCGAAAUUGAAUCACUCGCUAGCAAAUUAAACGACAAAGCACCCAAGGAGGGUGCGA ECNNV AAACAAACAAAACUGAAAUUGGAAAGAAUAGAAGCGAAAUUGAAUCACUCGCUAGUAAAUUAAACGACAAAGCACCCAAGGAGGGUGCGA DGNNV AAACAAACAAAACUGAAAUUGGAAAGAAUAGAAGCGAAAUUGAAUCACUCGCUAGCAAAUUAAACGACAAAGCACCCAAGGAGGGUGCGA WSNNV AAACAAACAAAACUGAAAUUGGAAAGAAUAGAAGCGAAAUUGAAUCACUCGCUAGCAAAUUAAACGACAAAGCACCCAAGGAGGGUGCGA TNV -----CAAAACCCAAAUUGCUCAAAACAAAAGCGAAAUUGACGCUAUCGCUAAUCAAUUAGACAACAAAGCACCCAAGGAGGGUGCCA

$$
* * * \quad * * * * * * \quad * * * * * * * * * * * * * * * * * * * * \quad * * * * * * * * * * * * * * * * * * * * * * * * * \quad * *
$$

Fig. 2. Multiple alignments of the UTRs of the 10 betanodavirus genomes (for virus names, see Table 2 legend). Nucleotide sequences of (A) the RNA1 5' UTRs, (B) the RNA1 3' UTRs, (C) the RNA2 5' UTRs, and (D) the RNA2 3' UTRs were compared using the ClustalW command. The lengths of the UTR sequences are denoted to the right. Initiation codons and stop codons are boxed. Asterisks indicate the nucleotide positions that were identical among all the viruses used 
TPKag93 UUGCUAUUGUUGGUACCAUUGACGGCGUACCUGCUACAAUUGAAGGCGGUUAUAUCGCUGGAUCUGUGCAGCGUGCCUGAUAAGGUGCCA JF Iwa98 UUGCUAUUGUUGGUACCAUUGACGGCGUACCUGGUACAGUUGACGGUGUUUACAUCGCGG---CUGUCUAGCGUGCUCGAUAUGGUGCCA SJNag93 UUGCUAUUGUUGGUACCAUUGACGGCGUACCUGGAACAGUUGACGGCGCUUACCUCGCCGAACCUGUCUAGCGUGCUUGAUACGGUGCCA SGWak97 UUGCUAUUGUUGGUACCCUUGACGGCGUACCGGCUACGCUUGAAGGCCUAUACACGGCUGGAAGCGCGCCGCGUGCUUAAUUGGGUGCCA GGNNV UUGCUAUUGUUGGUACCCUUGACGGCGUACCGGCUACGCUUGAAGGCCUAUACACGGCUGGAAGCGCGCCGCGUGCUUAAUUGGGUGCCA ECNNV UUGCUAUUGUUGGUACCCUUGACGGCGUACCGGCUACGCUUGAAGGCCUAUACACGGCUGGAAGCGCGCCGCGUGCUUAAUUGGGUGCCA DGNNV UUGCUAUUGUUGGUACCCUUGACGGCGUACCGGCUACGCUUGAAGGCCUAUACACGGCUGGAAGUGCGCCGCGUGCUUGAUUGGGUGCCA WSNNV UUGCUAUUGUUGGUACCCUUGACGGCGUACCGGCUACGCUUGAAGGCCUAUACACGGCUGGAAGCGCGCCGCGUGCUUAAUUGGGUGCCA TNV UUGCUAUCAUUGGUACCGUUGACGGCGUACCUGGAACUGUUGAUGGUGGCUACGUCACCACAAUAGUCUAGCGUGCUUGAUUUGGUACCA $* * * * * * \quad * * * * * * * * * * * * * * * * * * * * * * * * * * * * * \quad * \quad * \quad * * * * * * \quad * * * * * * * *$

TPKag93 GCUUUACCAGUAGUAUUCCCCCGCCGAGGAAAUCCUUCUUUGGGCUUGUUGGGUUACCGUUAGCUCCGCGCAGUGAGCACCACCGCCAUG JFIwa98 GCUUCACCAGUUGCAU-CCAACGCCGAGGAUUUCCCUCUUUGGGCUUGUUGGGUUACCGUUAGCUCCGCGUAGAGAGCACCACCGCCAUG SJNag93 GCUUCACCAGUCUUGU-CCAACGCCGAGGAUUUCCCUCUUUGGGCUUGUUGGGUUACCGUUAGCUCCGCGCAGUGAGCACCACCGCCAUG SGWak97 GUGGUACCAGUCGUAU-CCAACGCCGAGGAAGUCCCUCUUUGGGCU-GUUGGGUUACCGUUAGCUCCGCGUAGUGAGCACCACCGCCAUG GGNNV GUGGUACCAGUCGUAU-CCAACGCCGAGGAAGUCCCUCUUUGGGCU-GUUGGGUUACCGUUAGCUCCGCGCAGUGAGCACCACCGCCAUG ECNNV GUGGUACCAGUCGUAU-CCAACGCCGAGGAAAUCCCUCUUUGGGCU-GUUGGGUUACCGUUAGCUCCGCGCAGUGAGCACCACCGCCAUG DGNNV GUGGUACCAGUCGUAU-CCAACGCCGAGGAAUUCCCUCUUUGGGCU-GUUGGGUUACCGUUAGCUCCGCGCAGUGAGCACCACCGCCAUG WSNNV GUGGUACCAGUCGUAU-CCAACGCCGAGGAAAUCCCUCUUUGGGCU-GUUGGGUUACCGUUAGCUCCGCGCAGUGAGCACCACCGCCAUG TNV GUCUUACCAGUGGUAU-CCA-UGCCGAGGAUUUCCCCCCUUGGGCUUGUCGGAUUACCGUUAGCUCCGCGUAGUGAGCACCACCGCCAUG $* \quad * * * * * * \quad * * * \quad * * * * * * * * \quad * * * \quad * * * * * * * * * * * * * * * * * * * * * * * * * * * * * * * * * * * * * * * * * * * * * *$

TPKag93 UGGUUAAAUGGCCGCUGAUCGCUA-UUACAUUCGGCG (372)

JFIwa98 UGGUUAAAUGGCCGCUGAUCGCUAACUCUACUCGGCG (390)

SJNag 93 UGGUUAAAUGGCCGCUGAUCGCCA-CAUUACUCGGCG (371)

SGWak97 UGGUUAAAUGGCCGCUGAUCGCUU-CUCAACUCGGCG (391)

GGNNV UGGUUAAAUGGCCGCUGAUCGCUU-CUCAACUCGGC- (390)

ECNNV UGGUUAAAUGGCCGCUGAUCGCUU-CUCAACUCGGC- (390)

DGNNV UGGUUAAAUGGCCGCUGAUCGCUU-CUCAACUCGGC- (390)

WSNNV UGGUUAAAUGGCCGCUGAUCGCUU-CUCAACUCGGC- (390)

TNV UGGUUAAAUGGCCGCUGAUCGCU--CAUU--UCGGC- (366)

Fig. 2. (continued)

\section{Homology in RNA2 and CP among betanodaviruses}

Identities of the RNA2 sequences among the 9 betanodaviruses were 76 to $82 \%$, except among SGWak97, GGNNV, ECNNV, DGNNV, and white star snapper nervous necrosis virus (WSNNV), all of which belonged to the RGNNV genotype (Table 2, Fig. 1). Similarly, identities of the CP sequences were 77 to $81 \%$, except those between JFIwa98 and the 5 RGNNV isolates (86\%) and those among the 5 RGNNV isolates (98 to $100 \%$ ). The relatively high levels of shared CP sequence identities between JFIwa98 and the 5 RGNNV isolates support the previous report that BFNNV and RGNNV virions are serologically indistinguishable (Mori et al. 2003). Strikingly, the TNV RNA2 and CP were the least identical with those of any other viruses tested (Fig. 1). The identities of the 5' UTRs and 3' UTRs of RNA2s varied from 55 to $100 \%$ and from 80 to $100 \%$, respectively. The identities of JFIwa98 and the 5 RGNNV isolates were relatively high in the RNA2 5' UTRs (88\%) but low in the 3' UTRs (81 to $82 \%$ ). Similar relationships were observed between TNV and the 5 RGNNV iso- lates (Fig. 1). The multiple alignment of the 3' UTRs of the 9 betanodavirus RNA2s showed that the RNA2 3' UTRs of TPKag93, SJNag93, and TNV were 372, 371 , and $366 \mathrm{nt}$ in length, respectively, which were 18 to $24 \mathrm{nt}$ shorter than those of the other isolates belonging to BFNNV or RGNNV (Fig. 2D). These RNA2 3' UTRs of TPKag93, SJNag93, and TNV shared a consecutive $21 \mathrm{nt}$ gap in the alignment, suggesting that these 3 viruses are genetically close to each other. However, the nucleotide identities among these 3 sequences (81 to $86 \%$ ) were less than between TPKag93 and JFIwa98 (87\%), or between JFIwa98 and SJNag93 (88\%) (Fig. 1).

\section{Homology in RNA3 and Protein B2 among betanodaviruses}

Identities of RNA3 sequences among the betanodaviruses were 81 to $91 \%$, except among the isolates with the same genotypes: SGWak97, GGNNV, ECNNV, and DGNNV (95 to 98\%), and JFIwa98 and AHNV (99\%) (Fig. 1). In contrast, identities of 
Protein B2s varied from 73 to $90 \%$, excluding those among the isolates with the same genotypes (Fig. 1). Like the identities in RNA1 and Protein A observed in this study, the levels of identities in RNA3 and Protein B2 were relatively high between TPKag93 and the 2 BFNNV isolates (JFIwa98 and AHNV), though TPKag93 and the latter 2 viruses were classified into different genotypes. Protein B2 of SJNag93 looked unique because its sequence was the least identical with those of the other Protein B2s (73 to $78 \%$ ).

\section{CONCLUSION}

Betanodaviruses have been classified into 4 genotypes based on similarities in the partial RNA2 sequences (Nishizawa et al. 1997). Recently, TNV, which belonged to a putative fifth genotype, was detected in Norway (Johansen et al. 2004). However, only RGNNV and BFNNV are serologically indistinguishable using anti-SJNNV polyclonal antibody (Mori et al. 2003). That is, RGNNV and BFNNV are believed to be genetically close to each other. Although the virus numbers used in this study were limited in SJNNV, TPNNV, and BFNNV, we found further interesting relationships among the tested viruses. For example (1) TPKag93 and JFIwa98 shared the highest levels of identities in the RNA1 and Protein A sequences (Fig. 1), (2) SJNag93, TPKag93, and TNV shared similar lengths of the RNA2 3' UTRs, though the nucleotide sequences were not so homologous to each other (Figs. 1, 2D), (3) the 5' UTR of TPKag93 RNA1 was exceptionally long among the viruses tested (Fig. 2A), (4) the RNA1, RNA3, Protein A, and Protein B2 sequences of the RGNNV and BFNNV isolates were not so similar to each other compared with the high similarities in their RNA2 and CP sequences (Fig. 1). These results indicate that betanodaviruses have evolved through recombination events between RNA1 and RNA2, in addition to those within each viral segment and nucleotide substitution. We recently produced reassortants between SJNag93 and SGWak97, and confirmed that their infectivity was equivalent to that of the parental viruses (Iwamoto et al. 2004). To address the evolutionary process of betanodaviruses in detail, further analysis is required using complete genomic sequences from more isolates, especially of TPNNV and SJNNV.

Acknowledgements. This work was supported in part by grants-in-aid for Scientific Research (16380132, 16580151, 18580185) from the Ministry of Education, Culture, Sports, Science and Technology, Japan and a grant-in-aid for Scientific Research (18076) from the Ministry of Agriculture, Forestry and Fisheries of Japan.

\section{LITERATURE CITED}

Ball LA, Johnson KL (1998) Nodaviruses of insects. In: Miller LK, Ball LA (eds) The insect viruses. Plenum, New York, p 225-267

Chi SC, Shieh JR, Lin SJ (2003) Genetic and antigenic analysis of betanodaviruses isolated from aquatic organisms in Taiwan. Dis Aquat Org 55:221-228

Cutrín JM, Dopazo CP, Thiéry R, Leao P, Olveira JG, Barja JL, Bandín I (2007) Emergence of pathogenic betanodaviruses belonging to the SJNNV genogroup in farmed fish species from the Iberian Peninsula. J Fish Dis 30:225-232

Dasgupta R, Sgro JY (1989) Nucleotide sequences of three Nodaviruses RNA2s: the messengers for their coat protein precursors. Nucleic Acids Res 17:7525-7526

Dasmahapatra B, Dasgupta R, Ghosh A, Kaesberg P (1985) Structure of the black beetle virus genome and its functional implications. J Mol Biol 182:183-189

Furusawa R, Okinaka Y, Nakai T (2006) Betanodavirus infection in the freshwater model fish medaka (Oryzias latipes). J Gen Virol 87:2333-2339

Grotmol S, Nerland AH, Biering E, Totland GK, Nishizawa T (2000) Characterisation of the capsid protein gene from a nodavirus strain affecting the Atlantic halibut Hippoglossus hippoglossus and design of an optimal reversetranscriptase polymerase chain reaction (RT-PCR) detection assay. Dis Aquat Org 39:79-88

Harper TA (1994) Characterization of the proteins encoded from the nodaviral subgenomic RNA. PhD thesis, University of Wisconsin, Madison, WI

Hata N, Okinaka Y, Sakamoto T, Iwamoto T, Nakai T (2007) Upper temperature limits for the multiplication of betanodaviruses. Fish Pathol 42:225-228

Iwamoto T, Mori K, Arimoto M, Nakai T (1999) High permissivity of the fish cell line SSN-1 for piscine nodaviruses. Dis Aquat Org 39:37-47

Iwamoto T, Nakai T, Mori K, Arimoto M, Furusawa I (2000) Cloning of the fish cell line SSN-1 for piscine nodaviruses. Dis Aquat Org 43:81-89

Iwamoto T, Mise $\mathrm{K}$, Mori $\mathrm{K}$, Arimoto $\mathrm{M}$, Nakai $\mathrm{T}$, Okuno T (2001) Establishment of an infectious RNA transcription system for Striped jack nervous necrosis virus, the type species of the betanodaviruses. J Gen Virol 82:2653-2662

Iwamoto T, Okinaka Y, Mise K, Mori K, Arimoto M, Okuno T, Nakai $T$ (2004) Identification of host-specificity determinants in betanodaviruses using reassortants between striped jack nervous necrosis virus and 7band grouper nervous necrosis virus. J Virol 78:1256-1262

Iwamoto T, Mise K, Takeda A, Okinaka Y and others (2005) Characterization of Striped jack nervous necrosis virus subgenomic RNA3 and biological activities of its encoded Protein B2. J Gen Virol 86:2807-2816

Johansen R, Sommerset I, Tørud B, Korsnes K and others (2004) Characterization of nodavirus and viral encephalopathy and retinopathy in farmed turbot, Scophthalmus maximus (L.). J Fish Dis 27:591-601

Johnson KN, Ball LA (2003) Virions of Pariacoto virus contain a minor protein translated from the second AUG codon of the capsid protein open reading frame. J Gen Virol 84: $2847-2852$

Johnson KN, Zeddam JL, Ball LA (2000) Characterization and construction of functional cDNA clones of Pariacoto virus, the first Alphanodavirus isolated outside Australasia. J Virol 74:5123-5132

Johnson KN, Johnson KL, Dasgupta R, Gratsch T, Ball LA (2001) Comparisons among the larger genome segments 
of six nodaviruses and their encoded RNA replicases. J Gen Virol 82:1855-1866

Johnson KL, Price BD, Ball LA (2003) Recovery of infectivity from cDNA clones of nodamura virus and identification of small nonstructural proteins. Virology 305:436-451

Mori K, Mangyoku T, Iwamoto T, Arimoto M, Tanaka S, Nakai T (2003) Serological relationships among genotypic variants of betanodavirus. Dis Aquat Org 57:19-26

Munday BL, Kwang J, Moody N (2002) Betanodavirus infections of teleost fish: a review. J Fish Dis 25:127-142

Nishizawa T, Mori K, Nakai T, Furusawa I, Muroga K (1994) Polymerase chain reaction (PCR) amplification of RNA of striped jack nervous necrosis virus (SJNNV). Dis Aquat Org 18:103-107

Nishizawa T, Furuhashi M, Nagai T, Nakai T, Muroga K (1997) Genomic classification of fish nodaviruses by molecular phylogenetic analysis of the coat protein gene. Appl Environ Microbiol 63:1633-1636

Office International des Epizooties (OIE) (2006) Viral encephalopathy and retinopathy. In: Manual of diagnostic tests for aquatic animals, 5th edn. OIE, Paris, p 169-175

Editorial responsibility: Mark Crane,

Geelong, Victoria, Australia
Schneemann A, Ball LA, Delsert C, Johnson JE, Nishizawa T (2005) Family Nodaviridae. In: Fauquet CM, Mayo MA, Maniloff J, Desselberger U, Ball LA (eds) Virus taxonomy. Academic Press, San Diego, CA, p 865-872

Sommerset I, Nerland AH (2004) Complete sequence of RNA1 and subgenomic RNA3 of Atlantic halibut nodavirus (AHNV). Dis Aquat Org 58:117-125

Tan C, Huang B, Chang SF, Ngoh GH, Munday B, Chen SC, Kwang J (2001) Determination of the complete nucleotide sequences of RNA1 and RNA2 from greasy grouper (Epinephelus tauvina) nervous necrosis virus, Singapore strain. J Gen Virol 82:647-653

Thiéry R, Cozien J, de Boisséson C, Kerbart-Boscher S, Névarez L (2004) Genomic classification of new betanodavirus isolates by phylogenetic analysis of the coat protein gene suggests a low host-fish species specificity. J Gen Virol 85:3079-3087

Yoshikoshi K, Inoue K (1990) Viral nervous necrosis in hatchery-reared larvae and juveniles of Japanese parrotfish, Oplegnathus fasciatus (Temminck \& Schlegel). J Fish Dis 13:69-77

Submitted: November 19, 2007; Accepted: March 6, 2008 Proofs received from author(s): June 25, 2008 